\title{
Ingenhoff, Diana/Buhmann, Alexander: Public Diplomacy. Messung, Entstehung und Gestaltung von Landesimages
}

\author{
Köln: Herbert von Halem Verlag 2019. 276 Seiten. Preis: $€ 34,00$
}

\section{Olaf Hoffjann}

Online publiziert: 12. Juni 2020

(C) Der/die Autor(en) 2020

Das enorme Wachstum strategischer Kommunikation als Praxis- und Forschungsfeld lässt sich besonders eindrücklich beobachten an den vielen neuen Komposita, mit denen Praktiken zur Ansprache spezifischer Bezugsgruppen oder Organisationen benannt werden. Veröffentlichungen hierzu sind meist mit dem Hinweis verbunden, dass es die mit dem neuen Kompositum bezeichnete Praktik schon sehr lange gebe. Ähnlich verhält es sich mit „Public Diplomacy“, der sich Diana Ingenhoff und Alexander Buhmann in ihrem Buch widmen. Die Anfänge dieser Praktik verorten die Autoren in der sehr frühen Zivilisation. Dabei beziehen sie sich auf Michael Kunczik, der diese Bemühungen in seinem Buch „Die manipulierte Meinung“ 1990 noch als „Imagepflege von Staaten“ bezeichnete. Die Anlage beider Bücher veranschaulicht die empirisch-sozialwissenschaftliche Wende der Kommunikationswissenschaft im Allgemeinen und dieses Forschungsfeldes im Besonderen: Wo sich Michael Kunczik vor allem für ,,brisante und aufschlussreiche Beispiele“ interessierte, fokussieren Diana Ingenhoff und Alexander Buhmann jetzt auf empirische Aspekte zur Messung, Entstehung und Wirksamkeit von Länderimages sowie der Evaluation von Landeskommunikation. Diese vier Themen bilden die Struktur des Bandes. In jeweils einem Hauptkapitel werden hierzu bestehende Modelle und vorliegende Befunde ausgeführt. Diesen vier Hauptkapiteln ist eine kurze, siebenseitige Annäherung an das Forschungsfeld vorangestellt. Die Autoren definieren hier Public Diplomacy mit Cull (2009, S. 12) allgemein als , an international actor's attempt to manage the international environment through engagement with a foreign public“. In Abgrenzung zur traditionellen Diplomatie, bei der alle Aktivitäten hauptsächlich am politischen Zentrum eines Ziellandes ausgerichtet seien, nehme Public Diplomacy

Prof. Dr. O. Hoffjann ( $\square)$

Institut für Kommunikationswissenschaft, Otto-Friedrich-Universität Bamberg,

An der Weberei 5, 96045 Bamberg, Deutschland

E-Mail: olaf.hoffjann@uni-bamberg.de 
- insbesondere unter Berücksichtigung der Dynamik demokratischer Mediengesellschaften - die eigene und ausländische Öffentlichkeit in den Blick, um die öffentliche Meinung zu beeinflussen.

Im ersten Hauptteil stellen die Autoren die Vielzahl vorliegender Modelle zur Messung von Landesimages vor. Insgesamt 16 wirtschafts-, politik- und kommunikationswissenschaftliche sowie sozialpsychologische Modelle werden jeweils auf mehreren Seiten vorgestellt und kritisch reflektiert. Seinen Abschluss findet dieser Teil mit der Vorstellung des 4D- bzw. 5D-Modells, das beide Autoren in früheren Arbeiten entwickelt haben. Vielen dieser Ansätze ist gemeinsam, dass sie auf jährlicher Basis Länder-Rankings erstellen. Dabei zeige sich - so die Autoren resümierend -, dass Landesimages grundsätzlich eher stabil seien, allerdings ein politischer Führungs- und Richtungswechsel das Landesimage auch kurzfristig beeinflussen könne. Aktuelles Beispiel hierfür sei die Wahl Donald Trumps 2016, nach der die USA in allen Länder-Rankings um mehrere Ränge gefallen seien. So überzeugend die kenntnisreiche und detaillierte Darstellung der Ansätze für sich genommen ist, so hätte man sich zu Beginn dieses Kapitels eine längere Einführung zu den konzeptionellen und epistemologischen Grundlagen und später ein deutlicheres Herausarbeiten der Unterschiede zwischen den Modellen gewünscht. Und wenn die Autoren das eigene 4D- bzw. 5D-Modell insbesondere für seine „konzisen theoretischen Grundlage“ loben, hätte man hierzu gerne mehr erfahren.

Im zweiten Hauptteil steht die Entstehung von Landesimages im Mittelpunkt. Nach einer kurzen Skizzierung von Modellen zur Entstehung und Veränderung von Landesimages werden vor allem verschiedene Akteure und Stakeholdergruppen sowie Kanäle vorgestellt, bevor abschließend mit Großveranstaltungen sowie Krisen zwei Sondersituationen erläutert werden, die Landesimages kurzfristig verändern können. Bevor im abschließenden Hauptteil auf Basis bestehender Ansätze zum Kommunikationscontrolling die Evaluation von Landeskommunikation erläutert wird, stehen im dritten Hauptteil die Handlungsrelevanz und Wirksamkeit von Landesimages im Mittelpunkt. Spätestens hier wird deutlich, dass das Image eines Landes sehr unterschiedliche Entscheidungen beeinflussen kann. Dies beginnt in der Politik, wenn Deutschland und Großbritannien zu Beginn des Ersten Weltkriegs in den USA eine intensive Öffentlichkeitsarbeit betrieben haben, um die öffentliche Meinung und damit die Regierung für ihre Seite zu gewinnen. Es setzt sich im Marketing fort, wo das Herkunftsland als Qualitätssiegel Kaufentscheidungen beeinflusst - von Schweizer Uhren über italienische Schuhe bis hin zur Pariser Mode. Und es reicht schließlich bis zu Entscheidungen von Touristen, Fachkräften, Investoren und Studierenden, die im Buch erläutert werden.

Diese Bandbreite macht ein Problem des Bandes deutlich: Denn während es unbestritten ist, dass Public Diplomacy auf Landesimages zielt, sind umgekehrt Landesimages nicht nur in der Politik, sondern auch an vielen anderen Stellen handlungsrelevant. Hier zeigt sich, dass sich die Autoren primär für Landesimages und nur sekundär für Public Diplomacy interessieren. Dies wird auch daran deutlich, dass der im Untertitel genannte Aspekte der „Gestaltung“ und damit strategische sowie taktische Aspekte der Public Diplomacy wenig ausgeführt werden.

Dies ändert aber wenig am Wert des Buches: Denn sein zentraler Erkenntnisgewinn ist vor allem in den empirischen Aspekten zur Messung, Entstehung und 


\section{Wirksamkeit von Länderimages sowie der Evaluation von Landeskommunikation zu finden.}

Funding Open Access funding provided by Projekt DEAL.

Open Access Dieser Artikel wird unter der Creative Commons Namensnennung 4.0 International Lizenz veröffentlicht, welche die Nutzung, Vervielfältigung, Bearbeitung, Verbreitung und Wiedergabe in jeglichem Medium und Format erlaubt, sofern Sie den/die ursprünglichen Autor(en) und die Quelle ordnungsgemäß nennen, einen Link zur Creative Commons Lizenz beifügen und angeben, ob Änderungen vorgenommen wurden.

Die in diesem Artikel enthaltenen Bilder und sonstiges Drittmaterial unterliegen ebenfalls der genannten Creative Commons Lizenz, sofern sich aus der Abbildungslegende nichts anderes ergibt. Sofern das betreffende Material nicht unter der genannten Creative Commons Lizenz steht und die betreffende Handlung nicht nach gesetzlichen Vorschriften erlaubt ist, ist für die oben aufgeführten Weiterverwendungen des Materials die Einwilligung des jeweiligen Rechteinhabers einzuholen.

Weitere Details zur Lizenz entnehmen Sie bitte der Lizenzinformation auf http://creativecommons.org/ licenses/by/4.0/deed.de.

Prof. Dr. Olaf Hoffjann ist Professor für Kommunikationswissenschaft, insbesondere Organisationskommunikation und Öffentlichkeitsarbeit an der Otto-Friedrich-Universität Bamberg. 